\title{
A note on input-to-state stabilization for nonlinear sampled-data systems
}

\author{
Dragan Nešićc* and Dina S. Laila*
}

\begin{abstract}
We provide a framework for the design of $\mathcal{L}_{\infty}$ stabilizing controllers via approximate discretetime models for sampled-data nonlinear systems with disturbances. In particular, we present sufficient conditions under which a discrete-time controller that input-to-state stabilizes an approximate discrete-time model of a nonlinear plant with disturbances would also input-to-state stabilize (in an appropriate sense) the exact discrete-time plant model.
\end{abstract}

\section{Introduction}

A stumbling block in controller design for nonlinear sampled-data control systems is the absence of a good model for the design. Indeed, even if the continuous-time plant model is known, we can not in general compute the exact discrete-time model of the plant since this requires an explicit analytic solution of a nonlinear differential equation. This has motivated research on controller design via approximate discrete-time models for sampled-data nonlinear systems $[1,2,7]$. A drawback of these early results was their limited applicability: they investigate a particular class of plant models, a particular approximate discrete-time plant model (usually Euler) and a particular controller.

A more general framework for stabilization of disturbance-free sampled-data nonlinear systems via their approximate discrete-time models that is applicable to general plant models, controllers and approximate discrete-time models was first presented in $[8,10]$. In this paper, we generalize results in [10] by: (i) considering sampled-data nonlinear systems with disturbances; (ii) providing a framework for the design of input-to-state stabilizing (ISS) controllers based on approximate discrete-time plant models (for more details on ISS see $[6,12,13,14]$ ). In particular, we provide sufficient conditions on the continuous-time plant model, the controller and the approximate discrete-time model, which guarantee that if the controller input-to-state stabilizes the approximate discrete-time plant model it would also input-to-state stabilize the exact discrete-time plant model. Our results apply to dynamic controllers and our approach benefits from the results on numerical integration schemes in [15] and [3, 4].

*This research was supported by Australian Research Council under the Large Grants Scheme. The Department of Electrical and Electronic Engineering, The University of Melbourne, Parkville 3010, Victoria, Australia. E-mail: \{d.nesic, dsl\}@ee.mu.oz.au. 


\section{Preliminaries}

Sets of real and natural numbers (including 0 ) are denoted respectively as $\mathbb{R}$ and $\mathbb{N}$. For a given function $w: \mathbb{R}_{\geq 0} \rightarrow \mathbb{R}^{n}$, we use the following notation: $w_{T}[k]:=\{w(t): t \in[k T,(k+1) T]\}$ where $k \in \mathbb{N}$ and $T>0$ (in other words $w_{T}[k]$ is a piece of function $w(t)$ in the $k$-th sampling interval $[k T,(k+1) T]$ ); and $w(k)$ is the value of the function $w(\cdot)$ at $t=k T, k \in \mathbb{N}$. We denote the norms $\left\|w_{T}[k]\right\|_{\infty}=\sup _{\tau \in[k T,(k+1) T]}|w(\tau)|$ and $\|w\|_{\infty}:=\sup _{\tau \geq 0}|w(\tau)|$ and in the case when $w(\cdot)$ is a measurable function (in the Lebesgue sense) we use the essential supremum in the definitions. If $\|w\|_{\infty}<\infty$, then we write $w \in \mathcal{L}_{\infty}$. Consider a continuous-time nonlinear plant with disturbances:

$$
\dot{x}(t)=f(x(t), u(t), w(t)),
$$

where $x \in \mathbb{R}^{n_{x}}, u \in \mathbb{R}^{m}$ and $w \in \mathbb{R}^{p}$ are respectively the state, control input and exogenous disturbance. It is assumed that $f$ is locally Lipschitz and $f(0,0,0)=0$. We will consider two cases: $w(\cdot)$ are measurable functions (in the Lebesgue sense); and $w(\cdot)$ are continuously differentiable functions. We will always make precise which case we consider. The control is taken to be a piecewise constant signal $u(t)=u(k T)=: u(k), \forall t \in[k T,(k+1) T), k \in \mathbb{N}$, where $T>0$ is the sampling period. Also, we assume that some combination (output) or all of the states $(x(k):=x(k T))$ are available at sampling instant $k T, k \in \mathbb{N}$. The exact discrete-time model for the plant (1), which describes the plant behavior at sampling instants $k T$, is obtained by integrating the initial value problem

$$
\dot{x}(t)=f(x(t), u(k), w(t)),
$$

with given $w_{T}[k], u(k)$ and $x_{0}=x(k)$, over the sampling interval $[k T,(k+1) T]$. If we denote by $x(t)$ the solution of the initial value problem (2) at time $t$ with given $x_{0}=x(k), u(k)$ and $w_{T}[k]$, then the exact discrete-time model of (1) can be written as:

$$
x(k+1)=x(k)+\int_{k T}^{(k+1) T} f(x(\tau), u(k), w(\tau)) d \tau=: F_{T}^{e}\left(x(k), u(k), w_{T}[k]\right) .
$$

We refer to (3) as a functional difference equation since it depends on $w_{T}[k]$. We emphasize that $F_{T}^{e}$ is not known in most cases. Indeed, in order to compute $F_{T}^{e}$ we have to solve the initial value problem (2) analytically and this is usually impossible since $f$ in (1) is nonlinear. Hence, we will use an approximate discrete-time model of the plant to design a controller.

Different approximate discrete-time models can be obtained using different methods. For example, we may first assume that the disturbances $w(\cdot)$ are constant during sampling intervals, $w(t)=w(k), \forall t \in$ $[k T,(k+1) T]$ and then use a classical Runge-Kutta numerical integration scheme (such as Euler) for 
the initial value problem (2). In this case, the approximate discrete-time model can be written as

$$
x(k+1)=F_{T}^{a}(x(k), u(k), w(k)) .
$$

We refer to the approximate model (4) as an ordinary difference equation since $F_{T}^{a}$ does not depend on $w_{T}[k]$ but on $w(k)$. For instance, the Euler approximate model is $x(k+1)=x(k)+T f(x(k), u(k), w(k))$. Recently, numerical integration schemes for systems with measurable disturbances were considered in $[3,4]$. Using these numerical integration techniques we can obtain an approximate discrete-time model

$$
x(k+1)=F_{T}^{a}\left(x(k), u(k), w_{T}[k]\right),
$$

which is in general a functional difference equation. For instance, the simplest such approximate discrete-time model, which is analogous to Euler model, has the following form $x(k+1)=x(k)+$ $\int_{k T}^{(k+1) T} f(x(k), u(k), w(s)) d s$ (see [3]). Since we will consider semiglobal ISS (see Definition 2.2), we will think of $F_{T}^{e}$ and $F_{T}^{a}$ as being defined globally for all small $T$, even though the initial value problem (2) may exhibit finite escape times (see discussion on pg. 261 in [10]).

The sampling period $T$ is assumed to be a design parameter which can be arbitrarily assigned. Since we are dealing with a family of approximate discrete-time models $F_{T}^{a}$, parameterized by $T$, in order to achieve a certain objective we need in general to obtain a family of controllers, parameterized by $T$. We consider a family of dynamic feedback controllers

$$
\begin{aligned}
z(k+1) & =G_{T}(x(k), z(k)) \\
u(k) & =u_{T}(x(k), z(k)),
\end{aligned}
$$

where $z \in \mathbb{R}^{n_{z}}$. To shorten notation, we introduce $\tilde{x}:=\left(x^{T} z^{T}\right)^{T}, \tilde{x} \in \mathbb{R}^{n_{\tilde{x}}}$, where $n_{\tilde{x}}:=n_{x}+n_{z}$ and

$$
\mathcal{F}_{T}^{i}(\tilde{x}(k), \cdot):=\left(\begin{array}{c}
F_{T}^{i}\left(x(k), u_{T}(x(k), z(k)), \cdot\right) \\
G_{T}(x(k), z(k))
\end{array}\right) .
$$

The superscript $i$ may be either $e$ or $a$, where $e$ stands for exact model, $a$ for approximate model. We omit the superscript if we refer to a general model. The second argument of $\mathcal{F}_{T}^{i}(\tilde{x}, \cdot)$ (third argument of $\left.F_{T}^{i}\right)$ is either a vector $w(k)$ or a piece of function $w_{T}[k]$. Similar to [8], we define the following:

Definition 2.1 (Lyapunov-SP-ISS) The family of systems $\tilde{x}(k+1)=\mathcal{F}_{T}\left(\tilde{x}(k), w_{T}[k]\right)$ is Lyapunov semiglobally practically input-to-state stable (Lyapunov-SP-ISS) if there exist functions ${ }^{1} \alpha_{1}, \alpha_{2}, \alpha_{3} \in \mathcal{K}_{\infty}$ and $\tilde{\gamma} \in \mathcal{K}$, and for any strictly positive real numbers $\left(\Delta_{1}, \Delta_{2}, \delta_{1}, \delta_{2}\right)$ there exist strictly positive real numbers $T^{*}$ and $L$ such that for all $T \in\left(0, T^{*}\right)$ there exists a function $V_{T}: \mathbb{R}^{n_{\tilde{x}}} \rightarrow \mathbb{R}_{\geq 0}$ such that for all

\footnotetext{
${ }^{1}$ A function $\gamma: \mathbb{R}_{>0} \rightarrow \mathbb{R}_{>0}$ is of class- $\mathcal{K}$ if it is continuous, zero at zero and strictly increasing. It is of class- $\mathcal{K}_{\infty}$ if it is of class- $\mathcal{K}$ and is unbounded. A continuous function $\beta: \mathbb{R}_{>0} \times \mathbb{R}_{>0} \rightarrow \mathbb{R}_{>0}$ is of class- $\mathcal{K} \mathcal{L}$ if $\beta(\cdot, \tau)$ is of class- $\mathcal{K}$ for each $\tau \geq 0$ and $\beta(s, \cdot)$ is decreasing to zero for each $s>0$.
} 
$\tilde{x} \in \mathbb{R}^{n_{\tilde{x}}}$ with $|\tilde{x}| \leq \Delta_{1}$ and all $w \in \mathcal{L}_{\infty}$ with $\|w\|_{\infty} \leq \Delta_{2}$ the following holds:

$$
\begin{aligned}
\alpha_{1}(|\tilde{x}|) & \leq V_{T}(\tilde{x}) \leq \alpha_{2}(|\tilde{x}|) \\
\frac{1}{T}\left[V_{T}\left(\mathcal{F}_{T}\left(\tilde{x}, w_{T}\right)\right)-V_{T}(\tilde{x})\right] & \leq-\alpha_{3}(|\tilde{x}|)+\tilde{\gamma}\left(\left\|w_{T}\right\|_{\infty}\right)+\delta_{1},
\end{aligned}
$$

and, moreover, for all $x_{1}, x_{2}, z$ with $\left|\left(x_{1}^{T} z^{T}\right)^{T}\right|,\left|\left(x_{2}^{T} z^{T}\right)^{T}\right| \in\left[\delta_{2}, \Delta_{1}\right]$ and all $T \in\left(0, T^{*}\right)$, we have $\left|V_{T}\left(x_{1}, z\right)-V_{T}\left(x_{2}, z\right)\right| \leq L\left|x_{1}-x_{2}\right|$. The function $V_{T}$ is called an ISS-Lyapunov function for the family $\mathcal{F}_{T}$.

Remark 2.1 In the case when the family of parameterized closed-loop discrete-time nonlinear systems is an ordinary difference equation $\tilde{x}(k+1)=\mathcal{F}_{T}(\tilde{x}(k), w(k))$, the condition (9) is replaced by: for all $T \in\left(0, T^{*}\right)$, all $\tilde{x} \in \mathbb{R}^{n_{\tilde{x}}}$ with $|\tilde{x}| \leq \Delta_{1}$ and all $w \in \mathbb{R}^{p}$ with $|w| \leq \Delta_{2}$ we have

$$
\frac{1}{T}\left[V_{T}\left(\mathcal{F}_{T}(\tilde{x}, w)\right)-V_{T}(\tilde{x})\right] \leq-\alpha_{3}(|\tilde{x}|)+\tilde{\gamma}(|w|)+\delta_{1},
$$

and $V_{T}$ is called an ISS-Lyapunov function for the family $\mathcal{F}_{T}$.

The following definition is a semiglobal-practical version of the ISS property used in $[12,14]$ and we use it in the case when we consider measurable disturbances $w$.

Definition 2.2 (Semiglobal practical-ISS) The family of systems $\tilde{x}(k+1)=\mathcal{F}_{T}\left(\tilde{x}(k), w_{T}[k]\right)$ is semiglobally practically input-to-state stable (SP-ISS) if there exist $\beta \in \mathcal{K} \mathcal{L}$ and $\gamma \in \mathcal{K}_{\infty}$ such that for any strictly positive real numbers $\left(\Delta_{\tilde{x}}, \Delta_{w}, \delta\right)$ there exists $T^{*}>0$ such that for all $T \in\left(0, T^{*}\right),|\tilde{x}(0)| \leq \Delta_{\tilde{x}}$ and $w(\cdot)$ with $\|w\|_{\infty} \leq \Delta_{w}$, the solutions of the system satisfy $|\tilde{x}(k)| \leq \beta(|\tilde{x}(0)|, k T)+\gamma\left(\|w\|_{\infty}\right)+\delta$, $\forall k \in \mathbb{N}$.

The following semiglobal practical "ISS like property" was used in [9] and we use it when the disturbances are continuously differentiable.

Definition 2.3 (Semiglobal practical derivative ISS) The family of systems $\tilde{x}(k+1)=\mathcal{F}_{T}(\tilde{x}(k)$, $\left.w_{T}[k]\right)$ is semiglobally practically derivative input-to-state stable (SP-DISS) if there exist $\beta \in \mathcal{K} \mathcal{L}$ and $\gamma \in \mathcal{K}_{\infty}$ such that for any strictly positive real numbers $\left(\Delta_{\tilde{x}}, \Delta_{w}, \Delta_{\dot{w}}, \delta\right)$ there exists $T^{*}>0$ such that for all $T \in\left(0, T^{*}\right),|\tilde{x}(0)| \leq \Delta_{\tilde{x}}$ and all continuously differentiable $w(\cdot)$ such that $\|w\|_{\infty} \leq \Delta_{w}$ and $\|\dot{w}\|_{\infty} \leq \Delta_{\dot{w}}$, the solutions of the family $\mathcal{F}_{T}$ satisfy $|\tilde{x}(k)| \leq \beta(|\tilde{x}(0)|, k T)+\gamma\left(\|w\|_{\infty}\right)+\delta, \forall k \in \mathbb{N}$.

Note that a similar property to SP-ISS, called input to state practical stability (ISpS) was defined in $[5,13]$ when considering non-parameterized systems. 
Definition $2.4 u_{T}$ is said to be locally uniformly bounded if for any $\Delta_{\tilde{x}}>0$ there exist strictly positive numbers $T^{*}$ and $\Delta_{u}$ such that for all $T \in\left(0, T^{*}\right)$ and all $|\tilde{x}| \leq \Delta_{\tilde{x}}$ we have $\left|u_{T}(\tilde{x})\right| \leq \Delta_{u}$.

In order to prove our main results, we need to guarantee that the mismatch between $F_{T}^{e}$ and $F_{T}^{a}$ is small in some sense. We define two consistency properties, which will be used to limit the mismatch. Similar definitions can be found in numerical analysis literature (see Definition 3.4.2 in [15]) and recently in the context of sampled-data systems (see Definition 1 in [10], also Definition 2 in [8]). In the sequel we use the notation $x=x(k), u=u(k), w=w(k), w_{T}=w_{T}[k]$.

Definition 2.5 (One-step weak consistency) The family $F_{T}^{a}$ is said to be one-step weakly consistent with $F_{T}^{e}$ if given any strictly positive real numbers $\left(\Delta_{x}, \Delta_{u}, \Delta_{w}, \Delta_{\dot{w}}\right)$, there exist a function $\rho \in \mathcal{K}_{\infty}$ and $T^{*}>0$ such that, for all $T \in\left(0, T^{*}\right)$, all $x \in \mathbb{R}^{n_{x}}, u \in \mathbb{R}^{m}$ with $|x| \leq \Delta_{x},|u| \leq \Delta_{u}$ and functions $w(\cdot)$ that are continuously differentiable and satisfy $\left\|w_{T}\right\|_{\infty} \leq \Delta_{w}$ and $\left\|\dot{w}_{f}\right\|_{\infty} \leq \Delta_{\dot{w}}$, we have $\left|F_{T}^{e}-F_{T}^{a}\right| \leq T \rho(T)$.

Definition 2.6 (One-step strong consistency) The family $F_{T}^{a}$ is said to be one-step strongly consistent with $F_{T}^{e}$ if given any strictly positive real numbers $\left(\Delta_{x}, \Delta_{u}, \Delta_{w}\right)$, there exist a function $\rho \in \mathcal{K}_{\infty}$ and $T^{*}>0$ such that, for all $T \in\left(0, T^{*}\right)$, all $x \in \mathbb{R}^{n_{x}}, u \in \mathbb{R}^{m}, w \in \mathcal{L}_{\infty}$ with $|x| \leq \Delta_{x},|u| \leq \Delta_{u}$, $\left\|w_{T}\right\|_{\infty} \leq \Delta_{w}$, we have $\left|F_{T}^{e}-F_{T}^{a}\right| \leq T \rho(T)$.

Sufficient checkable conditions for one-step weak and strong consistency are given next (similar results for systems without disturbances are Lemma 1 in [8] and Lemma 1 in [10]).

Lemma 2.1 $F_{T}^{a}$ is one-step weakly consistent with $F_{T}^{e}$ if the following conditions hold: $1 . F_{T}^{a}$ is onestep weakly consistent with $F_{T}^{\text {Euler }}(x, u, w):=x+T f(x, u, w) ; 2$. given any strictly positive real numbers $\left(\Delta_{x}, \Delta_{u}, \Delta_{w}, \Delta_{\dot{w}}\right)$, there exist $\rho_{1} \in \mathcal{K}_{\infty}, \rho_{2} \in \mathcal{K}_{\infty}, T^{*}>0$, such that, for all $T \in\left(0, T^{*}\right)$, all $x_{1}, x_{2} \in \mathbb{R}^{n_{x}}$ with $\max \left\{\left|x_{1}\right|,\left|x_{2}\right|\right\} \leq \Delta_{x}$, all $u \in \mathbb{R}^{m}$ with $|u| \leq \Delta_{u}$ and all $w_{1}, w_{2} \in \mathbb{R}^{p}$ with $\max \left\{\left|w_{1}\right|,\left|w_{2}\right|\right\} \leq \Delta_{w}$, the following holds $\left|f\left(x_{1}, u, w_{1}\right)-f\left(x_{2}, u, w_{2}\right)\right| \leq \rho_{1}\left(\left|x_{1}-x_{2}\right|\right)+\rho_{2}\left(\left|w_{1}-w_{2}\right|\right)$.

Lemma 2.2 $F_{T}^{a}$ is one-step strongly consistent with $F_{T}^{e}$ if the following conditions hold: $1 . \quad F_{T}^{a}$ is one-step strongly consistent with $\tilde{F}_{T}^{E u l e r}\left(x, u, w_{T}\right):=x+\int_{k T}^{(k+1) T} f(x, u, w(s)) d s ;$ 2. given any strictly positive real numbers $\left(\Delta_{x}, \Delta_{u}, \Delta_{w}\right)$, there exist $\rho_{1} \in \mathcal{K}_{\infty}, T^{*}>0$, such that, for all $T \in\left(0, T^{*}\right)$ and for all $x_{1}, x_{2} \in \mathbb{R}^{n_{x}}$ with $\max \left\{\left|x_{1}\right|,\left|x_{2}\right|\right\} \leq \Delta_{x}$, all $u \in \mathbb{R}^{m}$ with $|u| \leq \Delta_{u}$ and all $w \in R^{p}$ with $|w| \leq \Delta_{w}$, the following holds $\left|f\left(x_{1}, u, w\right)-f\left(x_{2}, u, w\right)\right| \leq \rho_{1}\left(\left|x_{1}-x_{2}\right|\right)$.

Proofs of Lemmas 2.1 and 2.2 are similar to the proofs of Lemma 1 in [8] and Lemma 1 in [10]. 
Proof of Lemma 2.1: Let $\left(\Delta_{x}, \Delta_{u}, \Delta_{w}, \Delta_{\dot{w}}\right)$ be given. Using the numbers $\left(R_{x}, \Delta_{u}, \Delta_{w}, \Delta_{\dot{w}}\right)$, where $R_{x}=\Delta_{x}+1$, let the second condition of the lemma generate $T_{1}^{*}>0, \rho_{1} \in \mathcal{K}_{\infty}$ and $\rho_{2} \in \mathcal{K}_{\infty}$. Since $f$ is locally Lipschitz, it is locally bounded and there exists a number $M>0$ such that for all $|x| \leq R_{x}$, $|u| \leq \Delta_{u},|w|_{\infty} \leq \Delta_{w}$ we have $|f(x, u, w)| \leq M$. Let $T^{*}:=\min \left\{T_{1}^{*}, 1 / M\right\}$. It follows that, for each $|x| \leq \Delta_{x},\left\|w_{T}\right\|_{\infty} \leq \Delta_{w}$ and all $t \in[k T,(k+1) T]$, where $T \in\left(0, T^{*}\right)$, the solution $x(t)$ of

$$
\dot{x}(t)=f(x(t), u, w(t)), \quad x_{0}=x(k)=x
$$

satisfies $|x(t)| \leq R_{x}$ and $|x(t)-x| \leq M(t-k T) \leq M T$ and since $w(\cdot)$ is continuously differentiable by definition, we have $|w(t)-w(k)| \leq \Delta_{\dot{w}}(t-k T) \leq \Delta_{\dot{w}} T$, for all $t \in[k T,(k+1) T]$ and $T \in\left(0, T^{*}\right)$. It then follows from condition 2 of the lemma that, for all $|x| \leq \Delta_{x},|u| \leq \Delta_{u},\left\|w_{T}\right\|_{\infty} \leq \Delta_{w},\left\|\dot{w}_{f}[k]\right\|_{\infty} \leq \Delta_{\dot{w}}$, and all $T \in\left(0, T^{*}\right)$,

$$
\begin{aligned}
\left|\int_{k T}^{(k+1) T}[f(x(\tau), u, w(\tau))-f(x, u, w)] d \tau\right| & \leq \int_{k T}^{(k+1) T} \rho_{1}(|x(\tau)-x|) d \tau+\int_{k T}^{(k+1) T} \rho_{2}(|w(\tau)-w|) d \tau \\
& \leq T \rho_{1}(M T)+T \rho_{2}\left(\Delta_{\dot{w}} T\right) \leq T \tilde{\rho}(T),
\end{aligned}
$$

where $\tilde{\rho}(s):=\rho_{1}(M s)+\rho_{2}\left(\Delta_{\dot{w}} s\right)$ is a $\mathcal{K}_{\infty}$ function since $\rho_{1}$ and $\rho_{2}$ are $\mathcal{K}_{\infty}$. Since

$$
F_{T}^{e}=\underbrace{x+T f(x, u, w)}_{F_{T}^{\text {Euler }}}+\int_{k T}^{(k+1) T}[f(x(\tau), u, w(\tau))-f(x, u, w)] d \tau,
$$

the result follows from (12) and the first condition of the lemma, which implies the existence of $\tilde{\rho}_{1} \in \mathcal{K}_{\infty}$, such that $\left|F_{T}^{a}-F_{T}^{\text {Euler }}\right| \leq T \tilde{\rho}_{1}(T)$. Finally, by letting $\rho=\tilde{\rho}+\tilde{\rho}_{1}$ we prove that $F_{T}^{a}$ is one-step weakly consistent with $F_{T}^{e}$. Proof of Lemma 2.2 is omitted since it follows closely the proof of Lemma 2.1.

\section{Main Results}

In this section, we state and prove our main results (Theorems 3.1 and 3.2). The results specify conditions on the approximate model, the controller and the plant, which guarantee that the family of controllers $\left(G_{T}, u_{T}\right)$ that input-to-state stabilize $F_{T}^{a}$ would also input-to-state stabilize $F_{T}^{e}$ for sufficiently small $T$. We emphasize that our results are given for general approximate discrete-time models $F_{T}^{a}$ (not only for the Euler approximation). We remark that under certain mild conditions on the plant and the controller, our results can be extended to include inter-sample behavior, to conclude SP-ISS results for the closed-loop sampled-data systems (see results in [11]). Finally, an example is presented to illustrate our approach.

Theorem 3.1 Suppose that: (i) The family of approximate discrete-time models $\mathcal{F}_{T}^{a}(\tilde{x}, \cdot)$ is LyapunovSP-ISS (where either (9) or (10) holds); (ii) $F_{T}^{a}$ is one-step weakly consistent with $F_{T}^{e}$; (iii) $u_{T}$ is uniformly locally bounded. Then, the family of exact discrete-time models $\mathcal{F}_{T}^{e}\left(\tilde{x}, w_{T}\right)$ is SP-DISS. 
Theorem 3.2 Suppose that: (i) The family of approximate discrete-time models $\mathcal{F}_{T}^{a}\left(\tilde{x}, w_{T}\right)$ is LyapunovSP-ISS (where (9) holds); (ii) $F_{T}^{a}$ is one-step strongly consistent with $F_{T}^{e}$; (iii) $u_{T}$ is uniformly locally bounded. Then, the family of exact discrete-time models $\mathcal{F}_{T}^{e}\left(\tilde{x}, w_{T}\right)$ is SP-ISS.

The following lemmas are needed to complete proofs of both theorems. We prove only Lemma 3.1 for the case of ordinary difference equations (i.e., when (10) holds) and then comment on the changes in the proof for the case of functional difference equations (i.e., when (9) holds) and the proof of Lemma 3.2.

Lemma 3.1 If all conditions in Theorem 3.1 are satisfied, then there exist $\hat{\gamma} \in \mathcal{K}_{\infty}$ such that for any strictly positive numbers $\left(C_{\tilde{x}}, C_{w}, C_{\dot{w}}, \nu\right)$, there exists $T^{*}>0$ such that for all $T \in\left(0, T^{*}\right)$, we have

$$
\left\{\begin{array}{c}
|\tilde{x}| \leq C_{\tilde{x}},\|w\|_{\infty} \leq C_{w}, \quad\|\dot{w}\|_{\infty} \leq C_{\dot{w}} \\
\max \left\{V_{T}\left(\mathcal{F}_{T}^{e}\left(\tilde{x}, w_{T}\right)\right), V_{T}(\tilde{x})\right\} \geq \hat{\gamma}\left(\|w\|_{\infty}\right)+\nu
\end{array}\right\} \Longrightarrow \frac{V_{T}\left(\mathcal{F}_{T}^{e}\left(\tilde{x}, w_{T}\right)\right)-V_{T}(\tilde{x})}{T} \leq-\frac{1}{4} \alpha_{3}(|\tilde{x}|) .
$$

Lemma 3.2 If all conditions in Theorem 3.2 are satisfied, then there exist $\hat{\gamma} \in \mathcal{K}_{\infty}$ such that for any strictly positive numbers $\left(C_{\tilde{x}}, C_{w}, \nu\right)$, there exists $T^{*}>0$ such that for all $T \in\left(0, T^{*}\right)$, we have

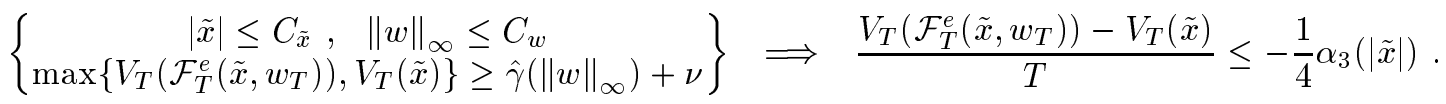

Proof of Lemma 3.1: First, we prove the following fact:

Fact 1: Suppose that for any strictly positive numbers $\left(\widetilde{\Delta}_{1}, \widetilde{\Delta}_{2}, \widetilde{\delta}_{1}\right)$ there exists $T_{w}^{*}>0$ such that for all $T \in\left(0, T_{w}^{*}\right),|\tilde{x}| \leq \widetilde{\Delta}_{1}$ and $|w| \leq \widetilde{\Delta}_{2}$ we have that (10) holds. Then, for any strictly positive numbers $\left(\Delta_{1}, \Delta_{2}, \Delta_{3}, \delta_{1}\right)$ there exists $T_{s}^{*}>0$ such that for all $T \in\left(0, T_{s}^{*}\right),|\tilde{x}| \leq \Delta_{1}$ and continuously differentiable disturbances with $\|w\|_{\infty} \leq \Delta_{2}$ and $\|\dot{w}\|_{\infty} \leq \Delta_{3}$ we have that

$$
\frac{V_{T}\left(\mathcal{F}_{T}(\tilde{x}, w)\right)-V_{T}(\tilde{x})}{T} \leq-\alpha_{3}(|\tilde{x}|)+\tilde{\gamma}\left(\left\|w_{T}\right\|_{\infty}\right)+\delta_{1} .
$$

Proof of Fact 1: Let $\left(\Delta_{1}, \Delta_{2}, \Delta_{3}, \delta_{1}\right)$ be given. Let $\widetilde{\delta}$ be such that $\sup _{s \in\left[0, \Delta_{2}\right]}|\tilde{\gamma}(s+\widetilde{\delta})-\tilde{\gamma}(s)| \leq \frac{\delta_{1}}{2}$. Let $\widetilde{\Delta}_{1}:=\Delta_{1}, \widetilde{\Delta}_{2}:=\Delta_{2}, \widetilde{\delta}_{1}:=\frac{\delta_{1}}{2}$ and let the numbers $\left(\widetilde{\Delta}_{1}, \widetilde{\Delta}_{2}, \widetilde{\delta}_{1}\right)$ generate $T_{w}^{*}>0$ from the condition of Fact 1. Let $T_{s}^{*}:=\min \left(T_{w}^{*}, \frac{\widetilde{\delta}}{\Delta_{3}}\right)$. Consider arbitrary $T \in\left(0, T_{s}^{*}\right),|\tilde{x}| \leq \Delta_{1}$ and any continuously differentiable disturbance with $\|w\|_{\infty} \leq \Delta_{2}$ and $\|\dot{w}\|_{\infty} \leq \Delta_{3}$. From the Mean Value Theorem and our choice of $T_{s}^{*}$ it follows that for all $t \in[k T,(k+1) T], k \in \mathbb{N}$ we have that $|w|=|w(k)| \leq$ $|w(t)-w(k T)|+|w(t)| \leq\left\|\dot{w}_{f}\right\|_{\infty}(t-k T)+\left\|w_{T}\right\|_{\infty} \leq \Delta_{3} T+\left\|w_{T}\right\|_{\infty} \leq \Delta_{3} T_{s}^{*}+\left\|w_{T}\right\|_{\infty} \leq \widetilde{\delta}+\left\|w_{T}\right\|_{\infty}$. Finally, using our definitions of $\widetilde{\delta}, \widetilde{\delta}_{1}$ we can write:

$$
\begin{aligned}
\frac{V_{T}\left(\mathcal{F}_{T}(\tilde{x}, w)\right)-V_{T}(\tilde{x})}{T} & \leq-\alpha_{3}(|\tilde{x}|)+\tilde{\gamma}(|w|)+\widetilde{\delta}_{1} \\
& =-\alpha_{3}(|\tilde{x}|)+\tilde{\gamma}\left(\left\|w_{T}\right\|_{\infty}\right)+\tilde{\gamma}(|w|)-\tilde{\gamma}\left(\left\|w_{T}\right\|_{\infty}\right)+\frac{\delta_{1}}{2} \\
& \leq-\alpha_{3}(|\tilde{x}|)+\tilde{\gamma}\left(\left\|w_{T}\right\|_{\infty}\right)+\tilde{\gamma}\left(\tilde{\delta}+\left\|w_{T}\right\|_{\infty}\right)-\tilde{\gamma}\left(\left\|w_{T}\right\|_{\infty}\right)+\frac{\delta_{1}}{2} \\
& \leq-\alpha_{3}(|\tilde{x}|)+\tilde{\gamma}\left(\left\|w_{T}\right\|_{\infty}\right)+\frac{\delta_{1}}{2}+\frac{\delta_{1}}{2}
\end{aligned}
$$


which completes the proof of the fact. Now we continue the proof of Lemma 3.1.

Suppose that all conditions in Theorem 3.1 (where (10) holds) are satisfied. Using Fact 1 it follows that all conditions in Theorem 3.1 (where (16) holds) are also satisfied. Let $\hat{\gamma}(s):=\alpha_{2} \circ \alpha_{3}^{-1}(4 \tilde{\gamma}(s)$ ). Let arbitrary strictly positive numbers $\left(C_{\tilde{x}}, C_{w}, C_{\dot{w}}, \nu\right)$ be given. Using $\left(C_{\tilde{x}}, C_{w}, C_{\dot{w}}, \nu\right)$, we define: $\epsilon:=\frac{1}{2} \alpha_{2}^{-1}\left(\frac{\nu}{2}\right) ; \delta_{1}:=\min \left\{\frac{1}{4} \alpha_{1}\left(\frac{\epsilon}{4}\right), \frac{1}{4} \alpha_{3} \circ \alpha_{2}^{-1}\left(\frac{1}{2} \alpha_{1}(\epsilon)\right)\right\} ; \delta_{2}:=\alpha_{2}^{-1}\left(\frac{1}{2} \alpha_{1}(\epsilon)\right) ;$ and $\Delta:=\alpha_{1}^{-1}\left(\alpha_{2}\left(C_{\tilde{x}}\right)+\right.$ $\left.\tilde{\gamma}\left(C_{w}\right)+\delta_{1}\right)+\epsilon$. Let the numbers $\left(\delta_{1}, \delta_{2}, \Delta, \epsilon\right)$ generate the numbers $T_{1}^{*}>0$ and $L>0$ from condition (i) of Theorem 3.1 (where (16) holds). Let $\Delta$ generate $\Delta_{u}>0$ and $T_{2}^{*}>0$ from condition (iii) of Theorem 3.1. Let the quadruple $\left(\Delta, \Delta_{u}, C_{w}, C_{\dot{w}}\right)$ generate $T_{3}^{*}>0$ and $\rho$ from condition (ii) of Theorem 3.1. Let strictly positive numbers $T_{4}^{*}, T_{5}^{*}, T_{6}^{*}, T_{7}^{*}$ be such that: $L \rho\left(T_{4}^{*}\right) \leq \frac{1}{4} \alpha_{3}\left(\delta_{2}\right)$; $T_{5}^{*} \rho\left(T_{5}^{*}\right) \leq \epsilon ; T_{6}^{*} \tilde{\gamma}\left(C_{w}\right) \leq \frac{1}{2} \alpha_{1}\left(\frac{1}{4} \epsilon\right) ;$ and $T_{7}^{*}\left(\frac{1}{4} \alpha_{3}\left(C_{\tilde{x}}\right)+\tilde{\gamma}\left(C_{w}\right)+\delta_{1}+L \rho\left(T_{7}^{*}\right)\right) \leq \frac{\nu}{2}$. Finally, we take $T^{*}=\min \left\{T_{1}^{*}, T_{2}^{*}, T_{3}^{*}, T_{4}^{*}, T_{5}^{*}, T_{6}^{*}, T_{7}^{*}, 1\right\}$.

In the calculations that follow, we consider arbitrary $T \in\left(0, T^{*}\right),|\tilde{x}| \leq C_{\tilde{x}},\|w\|_{\infty} \leq C_{w}$ and $\|\dot{w}\|_{\infty} \leq C_{\dot{w}}$. From (8), (9) and definition of $\Delta$ and the fact that $T^{*} \leq 1$, we have that

$\left|\mathcal{F}_{T}^{a}(\tilde{x}, w)\right| \leq \alpha_{1}^{-1}\left(V_{T}\left(\mathcal{F}_{T}^{a}(\tilde{x}, w)\right)\right) \leq \alpha_{1}^{-1}\left(V_{T}(\tilde{x})+T \tilde{\gamma}\left(\|w\|_{\infty}\right)+T \delta_{1}\right) \leq \alpha_{1}^{-1}\left(\alpha_{2}\left(C_{\tilde{x}}\right)+\tilde{\gamma}\left(C_{w}\right)+\delta_{1}\right)<\Delta$.

Using the condition (ii) of Theorem 3.1, inequality (18) and our choice of $\Delta$ and $T^{*}$ (in particular the choice of $T_{5}^{*}$ ), we can write:

$$
\begin{aligned}
\left|\mathcal{F}_{T}^{e}\left(\tilde{x}, w_{T}\right)\right| & \leq\left|\mathcal{F}_{T}^{a}(\tilde{x}, w)\right|+\left|\mathcal{F}_{T}^{e}\left(\tilde{x}, w_{T}\right)-\mathcal{F}_{T}^{a}(\tilde{x}, w)\right| \leq \alpha_{1}^{-1}\left(\alpha_{2}\left(C_{\tilde{x}}\right)+\tilde{\gamma}\left(C_{w}\right)+\delta_{1}\right)+T \rho(T) \\
& \leq \alpha_{1}^{-1}\left(\alpha_{2}\left(C_{\tilde{x}}\right)+\tilde{\gamma}\left(C_{w}\right)+\delta_{1}\right)+\epsilon=\Delta .
\end{aligned}
$$

Suppose that $V_{T}\left(\mathcal{F}_{T}^{e}\left(\tilde{x}, w_{T}\right)\right) \geq \hat{\gamma}\left(C_{w}\right)+\frac{\nu}{2}$. From (8), the definition of $\epsilon$ and the choice of $T^{*}$, we have

$$
\left|\mathcal{F}_{T}^{e}\left(\tilde{x}, w_{T}\right)\right| \geq \alpha_{2}^{-1}\left(\frac{\nu}{2}\right)=2 \epsilon>\epsilon,
$$

and then using the condition (ii) of Theorem 3.1 and our choice of $T_{5}^{*}$, we have

$$
\left|\mathcal{F}_{T}^{a}(\tilde{x}, w)\right| \geq-\left|\mathcal{F}_{T}^{e}\left(\tilde{x}, w_{T}\right)-\mathcal{F}_{T}^{a}(\tilde{x}, w)\right|+\left|\mathcal{F}_{T}^{e}\left(\tilde{x}, w_{T}\right)\right| \geq-T \rho(T)+\alpha_{2}^{-1}\left(\frac{\nu}{2}\right) \geq-\epsilon+2 \epsilon=\epsilon .
$$

From our choice of $T^{*} \leq 1, T_{6}^{*}, \delta_{1}$, and $\epsilon$ and using the inequality (16) it follows that:

$$
\begin{aligned}
\frac{1}{2} \alpha_{1}(\epsilon) & \leq \frac{1}{2} \alpha_{1}(\epsilon)+\frac{1}{2} \alpha_{1}(\epsilon)-\frac{1}{4} \alpha_{1}\left(\frac{\epsilon}{4}\right)-\frac{1}{4} \alpha_{1}\left(\frac{\epsilon}{4}\right) \leq \alpha_{1}(\epsilon)-T \tilde{\gamma}\left(C_{w}\right)-T \delta_{1} \\
& \leq \alpha_{1}\left(\left|\mathcal{F}_{T}^{a}(\tilde{x}, w)\right|\right)-T \tilde{\gamma}\left(C_{w}\right)-T \delta_{1} \leq V_{T}\left(\mathcal{F}_{T}^{a}(\tilde{x}, w)\right)-T \tilde{\gamma}\left(\|w\|_{\infty}\right)-T \delta_{1} \leq V_{T}(\tilde{x}) \leq \alpha_{2}(|\tilde{x}|)
\end{aligned}
$$

which implies:

$$
|\tilde{x}| \geq \alpha_{2}^{-1}\left(\frac{1}{2} \alpha_{1}(\epsilon)\right)=\delta_{2} .
$$


Note that $\delta_{2} \leq \epsilon$. From the conditions (i) and (ii) of Theorem 3.1 and from the choice of $T^{*}$ (in particular the choice of $T_{4}^{*}$ and $\left.T_{7}^{*}\right)$, the choice of $\delta_{1}$ and $\delta_{2}$ and using (18)-(23) we deduce that $V_{T}\left(\mathcal{F}_{T}^{e}\right) \geq \hat{\gamma}\left(C_{w}\right)+\frac{\nu}{2}$ implies

$$
\begin{aligned}
\hat{\gamma}\left(C_{w}\right)+\frac{\nu}{2} \leq & V_{T}\left(\mathcal{F}_{T}^{e}\right)-V_{T}(\tilde{x})+V_{T}(\tilde{x})+V_{T}\left(\mathcal{F}_{T}^{a}\right)-V_{T}\left(\mathcal{F}_{T}^{a}\right) \leq V_{T}\left(\mathcal{F}_{T}^{a}\right)-V_{T}(\tilde{x})+\left|V_{T}\left(\mathcal{F}_{T}^{e}\right)-V_{T}\left(\mathcal{F}_{T}^{a}\right)\right| \\
+V_{T}(\tilde{x}) \leq T \tilde{\gamma}\left(C_{w}\right)+T \delta_{1}+L T \rho(T)+V_{T}(\tilde{x}) & \leq \frac{\nu}{2}+V_{T}(\tilde{x}) .
\end{aligned}
$$

Hence, we can conclude that

$$
V_{T}\left(\mathcal{F}_{T}^{e}\right) \geq \hat{\gamma}\left(C_{w}\right)+\frac{\nu}{2} \Longrightarrow V_{T}(\tilde{x}) \geq \hat{\gamma}\left(C_{w}\right)
$$

Again using the conditions (i) and (ii) of Theorem 3.1 and from the choice of $T^{*}$ (in particular the choice of $T_{4}^{*}$ ), the choice of $\delta_{1}$ and $\delta_{2}$ and using (18)-(25) we can write:

$$
\begin{aligned}
V_{T}\left(\mathcal{F}_{T}^{e}\left(\tilde{x}, w_{T}\right)\right)-V_{T}(\tilde{x}) & \leq V_{T}\left(\mathcal{F}_{T}^{a}(\tilde{x}, w)\right)-V_{T}(\tilde{x})+\left|V_{T}\left(\mathcal{F}_{T}^{e}\left(\tilde{x}, w_{T}\right)\right)-V_{T}\left(\mathcal{F}_{T}^{a}(\tilde{x}, w)\right)\right| \\
& \leq-T \alpha_{3}(|\tilde{x}|)+T \tilde{\gamma}\left(\|w\|_{\infty}\right)+T \delta_{1}+L T \rho(T) \\
& \leq-\frac{T}{4} \alpha_{3}(|\tilde{x}|)-\frac{3 T}{4} \alpha_{3}(|\tilde{x}|)+T \tilde{\gamma}\left(C_{w}\right)+\frac{T}{4} \alpha_{3}\left(\delta_{2}\right)+\frac{T}{4} \alpha_{3}\left(\delta_{2}\right) \\
& \leq-\frac{T}{4} \alpha_{3}(|\tilde{x}|) \underbrace{-\frac{T}{4} \alpha_{3} \circ \alpha_{2}^{-1}\left(V_{T}(\tilde{x})\right)+T \tilde{\gamma}\left(C_{w}\right)}_{\leq 0} \underbrace{-\frac{T}{2} \alpha_{3}(|\tilde{x}|)+\frac{T}{2} \alpha_{3}\left(\delta_{2}\right)}_{\leq 0} \\
& \leq-\frac{T}{4} \alpha_{3}(|\tilde{x}|) .
\end{aligned}
$$

Suppose now that $V_{T}\left(\mathcal{F}_{T}^{e}\left(\tilde{x}, w_{T}\right)\right) \leq \hat{\gamma}\left(C_{w}\right)+\frac{\nu}{2}$ and $V_{T}(\tilde{x}) \geq \hat{\gamma}\left(C_{w}\right)+\nu$. From our choice of $T^{*}$ (in particular the choice of $T_{7}^{*}$ ), it follows that:

$$
V_{T}\left(\mathcal{F}_{T}^{e}\left(\tilde{x}, w_{T}\right)\right)-V_{T}(\tilde{x}) \leq \underbrace{\hat{\gamma}\left(C_{w}\right)+\frac{\nu}{2}-V_{T}(\tilde{x})+\frac{\nu}{2}}_{\leq 0}-\frac{\nu}{2} \leq-\frac{\nu}{2} \leq-\frac{T}{4} \alpha_{3}(|\tilde{x}|)
$$

which shows that (14) is valid, and this completes the proof of Lemma 3.1.

The proof of Lemma 3.1 for the case of functional difference equations and the proof of Lemma 3.3 follow the same steps as above except that we do not need to use Fact 1 since (9) holds. Also, in the case of functional difference equations of Lemma 3.1 we use one-step weak consistency and in the case of Lemma 3.3 we use one-step strong consistency. The next lemma is needed in proofs of Theorems 3.1 and 3.2 and it was proved as a part of the proof of Theorem 2 in [10].

Lemma 3.3 Let $\mathcal{W} \subset \mathcal{L}_{\infty}$ and let $\alpha_{1}, \alpha_{2}, \alpha_{3} \in \mathcal{K}_{\infty}$. Let strictly positive real numbers $(d, D)$ be such that $\alpha_{1}(D)>d$ and let $T^{*}>0$ be such that for any $T \in\left(0, T^{*}\right)$ there exists a function $V_{T}: \mathbb{R}^{n_{\tilde{x}}} \rightarrow \mathbb{R}_{\geq 0}$ such that for all $T \in\left(0, T^{*}\right)$ and all $\tilde{x} \in \mathbb{R}^{n_{\tilde{x}}}$ we have $\alpha_{1}(|\tilde{x}|) \leq V_{T}(\tilde{x}) \leq \alpha_{2}(|\tilde{x}|)$ and, moreover, for all $\tilde{x} \in \mathbb{R}^{n_{\tilde{x}}}$ with $\max \left\{V_{T}\left(\mathcal{F}_{T}\left(\tilde{x}, w_{T}\right)\right), V_{T}(\tilde{x})\right\} \geq d$ and $|\tilde{x}| \leq D$, all $w \in \mathcal{W}$ and all $T \in\left(0, T^{*}\right)$ the following holds $\frac{V_{T}\left(\mathcal{F}_{T}\left(\tilde{x}, w_{T}\right)\right)-V_{T}(\tilde{x})}{T} \leq-\frac{1}{4} \alpha_{3}(|\tilde{x}|)$. Then, there exist a function $\beta \in \mathcal{K} \mathcal{L}$ such that for all $T \in\left(0, T^{*}\right),|\tilde{x}(0)| \leq \alpha_{2}^{-1} \circ \alpha_{1}(D)$ and $w \in \mathcal{W}$ and all $k \in \mathbb{N}$ the solutions of the family of discrete-time models $\tilde{x}(k+1)=\mathcal{F}_{T}\left(\tilde{x}(k), w_{T}[k]\right)$ exist and satisfy $|\tilde{x}(k)| \leq \beta(|\tilde{x}(0)|, k T)+\alpha_{1}^{-1}(d)$. 
Proof of Theorem 3.1: Let arbitrary strictly positive real numbers $\left(\Delta_{\tilde{x}}, \Delta_{w}, \Delta_{\dot{w}}, \delta\right)$ be given and let all conditions in Theorem 3.1 hold. Let $\hat{\gamma} \in \mathcal{K}$ come from Lemma 3.1. We define $\left(C_{\tilde{x}}, C_{w}, C_{\dot{w}}, \nu\right)$ as: $C_{w}:=\Delta_{w}, C_{\dot{w}}:=\Delta_{\dot{w}}, \nu>0$ is such that $\sup _{s \in\left[0, \Delta_{w}\right]}\left[\alpha_{1}^{-1}(\hat{\gamma}(s)+\nu)-\alpha_{1}^{-1} \circ \hat{\gamma}(s)\right] \leq \delta$, and the number $C_{\tilde{x}}:=\max \left\{\alpha_{1}^{-1}\left(\hat{\gamma}\left(\Delta_{w}\right)+\nu\right)+1, \alpha_{1}^{-1} \circ \alpha_{2}\left(\Delta_{\tilde{x}}\right)\right\}$.

Using Lemma 3.1, let $\left(C_{\tilde{x}}, C_{w}, C_{\dot{w}}, \nu\right)$ generate $T^{*}>0$, such that (14) holds. Introduce $D:=C_{\tilde{x}}$ and $d:=\hat{\gamma}\left(\|w\|_{\infty}\right)+\nu$, and from the choice of $\left(C_{\tilde{x}}, C_{w}, C_{\dot{w}}, \nu\right)$ we have that $\alpha_{1}(D)>d$. Let $\mathcal{W}$ be a set of continuously differentiable functions defined as follows $\mathcal{W}:=\left\{w \in \mathcal{L}_{\infty} \mid\|w\|_{\infty} \leq C_{w},\|\dot{w}\|_{\infty} \leq C_{\dot{w}}\right\}$. With these definitions of $(D, d)$ and $\mathcal{W}$, together with (8), we have that all conditions of Lemma 3.3 hold. Hence, we can conclude that for all $T \in\left(0, T^{*}\right), \tilde{x}(0) \in \mathbb{R}^{n \tilde{x}},|\tilde{x}(0)| \leq \Delta_{\tilde{x}}$ and $w \in \mathcal{L}_{\infty}$ with $\|w\|_{\infty} \leq \Delta_{w},\|\dot{w}\|_{\infty} \leq \Delta_{\dot{w}}$ and all $k \geq 0$ we have that the solutions of $\mathcal{F}_{T}^{e}\left(\tilde{x}, w_{T}\right)$ exist and satisfy

$$
\begin{aligned}
|\tilde{x}(k)| & \leq \beta(|\tilde{x}(0)|, k T)+\alpha_{1}^{-1}(d) \leq \beta(|\tilde{x}(0)|, k T)+\alpha_{1}^{-1}\left(\hat{\gamma}\left(\|w\|_{\infty}\right)+\nu\right) \\
& \leq \beta(|\tilde{x}(0)|, k T)+\alpha_{1}^{-1} \circ \hat{\gamma}\left(\|w\|_{\infty}\right)+\delta=\beta(|\tilde{x}(0)|, k T)+\gamma\left(\|w\|_{\infty}\right)+\delta,
\end{aligned}
$$

where $\gamma(s):=\alpha_{1}^{-1} \circ \hat{\gamma}(s)$. This completes the proof of Theorem 3.1. The proof of Theorem 3.2 is omitted since it follows closely the proof of Theorem 3.1.

We illustrate below our results via an example.

Example 3.1 Consider the scalar continuous-time plant $\dot{x}(t)=x^{3}(t)+u(t)+w(t)$ and its approximate discrete-time model $x(k+1)=x(k)+T\left(x^{3}(k)+u(k)\right)+\int_{k T}^{(k+1) T} w(s) d s=: F_{T}^{a}\left(x(k), u(k), w_{T}[k]\right)$, which can be obtained from numerical integration schemes described in [3]. The following three controllers:

$$
\begin{aligned}
& u_{T}^{1}(x)=-x^{3}-x \\
& u_{T}^{2}(x)=-x^{3}-x-T x \\
& u_{T}^{3}(x)=-\frac{1}{2 T}\left[1+2 T x^{2}-\sqrt{1-4 T}\right] x
\end{aligned}
$$

can be shown to yield respectively the following three dissipation inequalities with $V(x)=\frac{1}{2} x^{2}$ :

$$
\begin{aligned}
& \frac{V\left(F_{T}^{a}\left(x, u_{T}^{1}(x), w_{T}\right)\right)-V(x)}{V\left(F_{T}^{a}\left(x, u_{T}^{2}(x), w_{T}\right)\right)-V(x)} \leq-\frac{1}{2} x^{2}+\frac{1}{2}\left\|w_{T}\right\|_{\infty}^{2}+T\left\|w_{T}\right\|_{\infty}^{2}+T x^{2} \\
& \frac{V\left(F_{T}^{a}\left(x, u_{T}^{3}(x), w_{T}\right)\right)-V(x)}{T} \leq-\frac{1}{2} x^{2}+\frac{1}{2}\left\|w_{T}\right\|_{\infty}^{2}+\left(T+\frac{T^{2}}{2}\right)\left\|w_{T}\right\|_{\infty}^{2}+\left(T+\frac{T^{2}}{2}+\frac{T^{3}}{2}\right) x^{2} \\
& \frac{w_{T}}{T}\left\|_{\infty}^{2}+T\right\| w_{T} \|_{\infty}^{2} .
\end{aligned}
$$

From our choice of $V(x)$ and (30) it follows that the approximate discrete-time model with any of the controllers (29) is Lyapunov SP-ISS. Moreover, since the approximate discrete-time model is the same as $\tilde{F}_{T}^{\text {Euler }}$ in the first condition of Lemma 2.2, it follows that $F_{T}^{a}$ is one-step strongly consistent with $F_{T}^{e}$. Finally, all of the controllers in (29) are locally uniformly bounded (for $u_{T}^{1}$ and $u_{T}^{2}$ this is obvious and for $u_{T}^{3}$ this can be seen by using the Taylor series expansion $\sqrt{1-4 T}=1-2 T+O\left(T^{2}\right)$ ). Therefore, for $F_{T}^{a}, V(x)$ and any controller in (29) we have that all conditions of Theorem 3.2 hold. Hence, we can conclude using Theorem 3.2 that each of controllers (29) semiglobally practically input-to-state stabilizes the exact discrete-time plant model. 


\begin{tabular}{||c|c|c|c||}
\hline \multirow{2}{*}{$\mathrm{T}[\mathrm{s}]$} & \multicolumn{3}{|c||}{ ROA estimate } \\
\cline { 2 - 4 } & $u_{T}^{1}(k)$ & $u_{T}^{2}(k)$ & $u_{T}^{3}(k)$ \\
\hline \hline 0.25 & {$[-2.99,2.99]$} & {$[-2.90,2.90]$} & {$[-2.66,2.66]$} \\
0.15 & {$[-4.10,4.10]$} & {$[-4.04,4.04]$} & {$[-4.01,4.01]$} \\
0.05 & {$[-7.78,7.78]$} & {$[-7.75,7.75]$} & {$[-7.75,7.75]$} \\
0.001 & {$[-67.81,67.81]$} & {$[-67.81,67.81]$} & {$[-67.81,67.81]$} \\
\hline
\end{tabular}

Table 1: ROAs in disturbance free case.

\begin{tabular}{||c|c|c|c|c||}
\hline \multirow{2}{*}{$\mathrm{T}[\mathrm{s}]$} & \multirow{2}{*}{$x_{\circ}$} & \multicolumn{3}{|c||}{ Amplitude of disturbance } \\
\cline { 3 - 5 } & & $u_{T}^{1}(k)$ & $u_{T}^{2}(k)$ & $u_{T}^{3}(k)$ \\
\hline \hline 0.25 & 2.66 & 2.50 & 3.04 & 4.37 \\
0.15 & 4.01 & 3.48 & 3.95 & 4.20 \\
0.05 & 7.75 & 6.84 & 7.12 & 7.15 \\
0.001 & 67.81 & 63.62 & 63.70 & 63.70 \\
\hline
\end{tabular}

Table 2: Performance with a disturbance

We applied the controllers (29) via a sampler and zero order hold to the continuous-time plant model and compared the performance of the three controllers via simulations in SIMULINK ${ }^{2}$. Note that the controller $u_{T}^{1}(x)$ may be obtained using a continuous-time design (obtain $\dot{V} \leq-\frac{1}{2} x^{2}+\frac{1}{2} w^{2}$ for the continuous-time closed-loop) and controller discretization. In Table 1 we estimated regions of attraction (ROA) of the closed-loop sampled-data system with controllers (29) for different sampling periods. The controller $u_{T}^{1}$ gives the largest ROA for all tested sampling periods. In Table 2 we summarize simulations for different sampling periods and fixed initial states with a sinusoidal disturbance of frequency $1 \frac{\mathrm{rad}}{\mathrm{sec}}$. The values of amplitude of the sinusoidal disturbance recorded in Table 2 are the largest values for which solutions of the sampled-data closed-loop system stay bounded. It is obvious that the controller $u_{T}^{3}$ is the most robust with respect to the test disturbance for all tested sampling periods. Similar observations were obtained for other initial states and disturbances that are not presented in Table 2. From Tables 1 and 2 we see that the performance of all controllers (29) becomes very similar for small sampling periods which can be expected since the dissipation inequalities in (30) differ only in terms of order T, which can be made arbitrarily small on compact sets by reducing $T$. Difference in performance of controllers (29) is more pronounced for larger sampling periods (see Tables 1 and 2).

\section{References}

[1] D. Dochain and G. Bastin, "Adaptive identification and control algorithms for nonlinear bacterial growth systems," Automatica, vol. 20, pp. 621-634, 1984.

[2] G. C. Goodwin, B. McInnis and R. S. Long, "Adaptive control algorithm for waste water treatment and pH neutralization," Optim. Contr. Appl. Meth., vol. 3, pp. 443-459, 1982.

[3] L. Grüne and P.E. Kloeden, "Higher order numerical schemes for affinely controlled nonlinear systems," Numerische Mathematik, vol. 89, pp. 669-690, 2001.

\footnotetext{
${ }^{2}$ We used the following parameters in simulations: variable step size; ode- 45 ; relative tolerance $10^{-3}$, absolute tolerance $10^{-6}$; max step size auto; initial step size auto.
} 
[4] R. Ferretti, "Higher order approximations of linear control systems via Runge Kutta schemes," Computing, vol. 58, pp. 351-364, 1997.

[5] Z.P. Jiang, A.R. Teel, and L. Praly, "Small gain theorem for ISS systems and applications," Math. Control, Signals and Systems, vol. 7, pp. 95-120, 1994.

[6] M. Krstić, I. Kanellakopoulos and P. V. Kokotović, Nonlinear and adaptive control design, J. Wiley \& Sons: New York, 1995..

[7] I. M. Y. Mareels, H. B. Penfold and R. J. Evans, "Controlling nonlinear time varying systems via Euler approximations," Automatica, vol. 28, pp. 681-696, 1992.

[8] D. Nešić and A. R. Teel, "Set stabilization of sampled-data nonlinear differential inclusions via their approximate discrete-time models," Proc. 39th Conf. on Decis. and Control, Sydney, pp. 2112-2117, 2000.

[9] D. Nešić and A. R. Teel, "Input-to-state stability for nonlinear time-varying systems via averaging," Math. Control, Signals and Systems, vol. 14, pp. 257-280, 2001.

[10] D. Nešić, A. R. Teel and P. Kokotović, "Sufficient conditions for stabilization of sampled-data nonlinear systems via discrete-time approximations," Syst. Contr. Lett., vol. 38, pp. 259-270, 1999.

[11] D. Nešić, A. R. Teel and E. Sontag, "Formulas relating $\mathcal{K} \mathcal{L}$ stability estimates of discrete-time and sampled-data nonlinear systems," Syst. Contr. Lett., vol. 38, pp. 49-60, 1999.

[12] E. D. Sontag, "Smooth stabilization implies coprime factorization," IEEE Trans. Automat. Contr., vol. 34, pp. 435-443, 1989.

[13] E.D. Sontag and Y. Wang, "On characterizations of the input-to-state stability with respect to compact sets," Proceedings of IFAC Non-Linear Control Systems Design Symposium, (NOLCOS '95), pp. 226-231, Tahoe City, CA, June 1995.

[14] E.D. Sontag, "The ISS philosophy as a unifying framework for stability-like behaviour," in Nonlinear Control in the Year 2000, (Eds. A.Isidori and F.Lamnabhi-Lagarrigue and W.Respondek), Springer-Verlag, Berlin, 2000, pp. 443-468.

[15] A. M. Stuart and A. R. Humphries, Dynamical Systems and Numerical Analysis. Cambridge University Press: Cambridge, 1996. 